\title{
Location estimation method for unknown signal source ${ }^{\dagger}$
}

\author{
Satoru Shimizu ${ }^{1, \text { a) }}$, Takuya Kurihara ${ }^{1}$, Kazuto Yano ${ }^{1}$, \\ and Yoshinori Suzuki ${ }^{1}$ \\ ${ }^{1}$ Advanced Telecommunications Research Institute International, \\ 2-2-2 Hikaridai, Keihanna Science City, Kyoto, 619-0288 Japan \\ a)dr.shimizu@atr.jp
}

Abstract: In this letter, we propose a location estimation method for an unknown signal source. We considered this problem as nonlinear optimization one and found an effective solution. First, RSSI samples associated with location information are obtained. This problem is solved by finding the position and effective radiated power at which the error between the measured RSSI and the RSSI estimated from an assumed propagation loss equation is minimized. The validity of this method was clarified by numerical evaluation.

Keywords: RSSI, location estimation, nonlinear optimization Classification: Sensing

\section{References}

[1] S. Shimizu, T. Kurihara, K. Yano, and Y. Suzuki, "Research summary of optimum communication method corresponding to radio wave environment" Proc. IEICE Gen. Conf. 2019, B-17-4, 2019.

[2] D. Adamy, "Location of communication emitters" in Tactical Battlefield Communications Electronic Warfare (Japanese version), pp. 190-219, TDU Press, Tokyo, 2015. ISBN 978-4-501-33100-9.

[3] M. Bshara, U. Orguner, F. Gustafsson, and L.V. Biesen, "Fingerprinting localization in wireless networks based on received-signal-strength measurements: a case study on WiMAX networks," IEEE Trans. Veh. Technol., vol. 59, no. 1, pp. 283-294, Jan. 2010. DOI: 10.1109/TVT.2009.2030504

[4] M. Ohtani, H. Iwai, and H. Sasaoka, "Evaluation of measurement interval of location fingerprint in location estimation method based on propagation characteristics," IEICE Technical Report, AP2014-30, pp. 63-66, May 2014.

[5] S. Shimizu, T. Kurihara, M. Usui, K. Yano, and Y. Suzuki, "Analytical method of location estimation for unknown signal source — basic study and development of radio monitoring node "Radio Catcher"-,, Proc. 6th International Workshop on Smart Wireless Communications (SmartCom 2019), Nov. 2019.

[6] S. Shimizu, T. Kurihara, K. Yano, and Y. Suzuki, "Analytical method of location estimation for unknown signal source — basic study —," IEICE Technical Report, SR2019-3, pp. 17-21, May 2019.

[7] T. Kurihara, S. Shimizu, K. Yano, and Y. Suzuki, "Analytical method of location estimation for unknown signal source - experimental basic evaluation-," IEICE Technical Report, SR2019-3, pp. 23-30, May 2019.

\footnotetext{
${ }^{\dagger}$ This letter has been partly presented in $[5,6,7]$.
} 


\section{Introduction}

In recent years, with the spread of IoT devices, various wireless devices have increased around us. Further, the operating frequency of the electronic circuit is increased, and the housing is often made of resin, so that high-frequency noise is easily generated. We are conducting research and development for optimal communication even in such an environment [1]. In the research, we conducted field measurements at many sites. As a result, we observed many radio noises whose signal sources were unknown. The demand for locating these sources is expected to increase in the future.

There are high performance methods to find the arrival direction of a signal using time or phase difference between antennas [2]. However, since extremely high time accuracy among multiple antenna is required, a large cost is required to construct the system by this method.

We studied a location estimation method for an unknown signal source using RSSI, that was relatively easy to measure. RSSI often fluctuates greatly even with a small change in location due to the influence of multipath. To overcome this problem, past methods using RSSI often found locations by comparing them with values measured in advance [3, 4]. However, those required prior measurement.

Here, we thought that the effect of multipath can be mitigated by acquiring a large amount of RSSI while moving the measuring point. Furthermore, we tried to solve the problem to estimate the signal source by non-linear optimization. As a result, it was found that the location of the signal source can be estimated by a simple optimization method such as the steepest descent method which gradually converge to the correct values using the update vector $[5,6,7]$.

Our previous work did not show a concrete calculation of the update vector. In reference [7], an evaluation result brought by an optimization solver built in a simulation tool was shown. This letter describes the basic principle of the location estimation method. The update vector assuming free space loss is also shown in the description. The simulation results indicate that the signal source location and EIRP can be estimated by this method using the update vector newly described in this letter.

\section{Estimation algorithm}

Let the position of the unknown signal source and the effective radiated power be $(\hat{x}, \hat{y}, \hat{z}, \hat{p})$. These values are all unknown and to be estimated. The coordinates and RSSI of position $i$ are described as $\left(x_{i}, y_{i}, z_{i}, r_{i}\right)$. Coordinates can be grasped by GPS, and RSSI can be measured by a receiver or spectrum analyzer. It is easy to acquire a large amount of RSSI with measured coordinates while moving the receiving point.

An estimated RSSI $\hat{r}_{i}$ is represented by a function with the parameters of the antenna gain connected to the radio monitoring node $G_{r}$, the wave length $\lambda$, estimated EIRP $\hat{p}$, and estimated distance $\hat{d}_{i}$ as a follow.

$$
\hat{r}_{i}=S\left(G_{r}, \lambda, \hat{p}, \hat{d}_{i}\right)
$$

where $\hat{d}_{i}$ is expressed by

$$
\hat{d}_{i}^{2}=\left(\hat{x}_{i}-x_{i}\right)^{2}+\left(\hat{y}_{i}-y_{i}\right)^{2}+\left(\hat{z}_{i}-z_{i}\right)^{2} .
$$


The value corresponding to the antenna gain of the unknown signal source is included in the transmitted power $\hat{p}$ as the effective radiated power.

An error function $f(\hat{x}, \hat{y}, \hat{z}, \hat{p})$ can be derived from the difference between the estimated received power $\hat{r}_{i}$ and the actually measured received power $r_{i}$ as

$$
f(\hat{x}, \hat{y}, \hat{z}, \hat{p})=\sum_{i=1}^{N} w_{i}\left(r_{i}-\hat{r}_{i}\right)^{2}
$$

where $w_{i}$ is a weight that can be changed depending on the reliability of measured RSSI. The location estimation is replaced by a nonlinear optimization problem that minimizes this error function.

At the point where Eq. (3) is minimized, the partial differentiation at each variable is zero.

$$
\nabla f(\hat{x}, \hat{y}, \hat{z}, \hat{p})=\left[\begin{array}{l}
\frac{\partial f(\hat{x}, \hat{y}, \hat{z}, \hat{p})}{\partial \hat{x}} \\
\frac{\partial f(\hat{x}, \hat{y}, \hat{z}, \hat{p})}{\partial \hat{y}} \\
\frac{\partial f(\hat{x}, \hat{y}, \hat{z}, \hat{p})}{\partial \hat{z}} \\
\frac{\partial f(\hat{x}, \hat{y}, \hat{z}, \hat{p})}{\partial \hat{p}}
\end{array}\right]=\left[\begin{array}{l}
0 \\
0 \\
0 \\
0
\end{array}\right] .
$$

Various algorithms can be available to solve this. One simple way is a steepest descent method. Then, coordinates and RSSI are obtained by a following equation.

$$
\left[\begin{array}{l}
\hat{x}_{k+1} \\
\hat{y}_{k+1} \\
\hat{z}_{k+1} \\
\hat{p}_{k+1}
\end{array}\right]=\left[\begin{array}{l}
\hat{x}_{k} \\
\hat{y}_{k} \\
\hat{z}_{k} \\
\hat{p}_{k}
\end{array}\right]-\alpha_{k} \nabla f\left(\hat{x}_{k}, \hat{y}_{k}, \hat{z}_{k}, \hat{p}_{k}\right)
$$

where $\alpha_{k}$ is a step size. If the update becomes smaller, it can be determined as a convergence point. Of course, initial values should be selected appropriately.

If the measurement environment is a free space, Eq. (1) is described as a following equation.

$$
\hat{r}_{i}=\hat{p} G_{r}\left(\frac{\lambda}{4 \pi \hat{d}_{i}}\right)^{2} .
$$

Then, the update vector $\nabla f\left(\hat{x}_{k}, \hat{y}_{k}, \hat{z}_{k}, \hat{p}_{k}\right)$ of Eq. (5) is given by

$$
\left[\begin{array}{l}
\frac{\partial f\left(\hat{x}_{k}, \hat{y}_{k}, \hat{z}_{k}, \hat{p}_{k}\right)}{\partial \hat{x}_{k}} \\
\frac{\partial f\left(\hat{x}_{k}, \hat{y}_{k}, \hat{z}_{k}, \hat{p}_{k}\right)}{\partial \hat{y}_{k}} \\
\frac{\partial f\left(\hat{x}_{k}, \hat{y}_{k}, \hat{z}_{k}, \hat{p}_{k}\right)}{\partial \hat{z}_{k}} \\
\frac{\partial f\left(\hat{x}_{k}, \hat{y}_{k}, \hat{z}_{k}, \hat{p}_{k}\right)}{\partial \hat{p}_{k}}
\end{array}\right]=\left[\begin{array}{c}
4 \hat{p} G_{r} \sum_{i=1}^{N} w_{i}\left(\frac{\lambda}{4 \pi \hat{d}_{i}}\right)^{2} \frac{\left(\hat{x}_{k}-x_{i}\right)}{\hat{d}_{i}^{2}}\left(r_{i}-\hat{r}_{i}\right) \\
4 \hat{p} G_{r} \sum_{i=1}^{N} w_{i}\left(\frac{\lambda}{4 \pi \hat{d}_{i}}\right)^{2} \frac{\left(\hat{y}_{k}-y_{i}\right)}{\hat{d}_{i}^{2}}\left(r_{i}-\hat{r}_{i}\right) \\
4 \hat{p} G_{r} \sum_{i=1}^{N} w_{i}\left(\frac{\lambda}{4 \pi \hat{d}_{i}}\right)^{2} \frac{\left(\hat{z}_{k}-z_{i}\right)}{\hat{d}_{i}^{2}}\left(r_{i}-\hat{r}_{i}\right) \\
-2 G_{r} \sum_{i=1}^{N} w_{i}\left(\frac{\lambda}{4 \pi \hat{d}_{i}}\right)^{2}\left(r_{i}-\hat{r}_{i}\right)
\end{array}\right] .
$$




\section{Simulation results}

A simple two-dimensional simulation was conducted for verification. A transmitter with a transmitting power 1 is placed at $x=y=1$. Antenna gain and wave length are set to $G_{r}=\lambda=1$. There are 21 measurement points. They are located $y=10$, and $x$ is 21 points from -10 to 10 in 1 step. An unknown signal position and measurement points are shown in Fig. 1. The propagation loss were assumed to be free space loss.

The simulation results are shown in Fig. 2. Initial values are $\hat{x}=\hat{y}=0, \hat{p}=0.5$, and step size $\alpha_{k}$ was $10^{7}$.

We can find that all variables converge to the correct values from Fig. 2. $\hat{p}$ started from 0.5 , overshot to 1.2 , and then converged to 1 . $\hat{x}$ converged less than 500 iterations. The slow convergence of $\hat{y}$ is due to small changes in the positions of the measurement points in the $y$-axis direction. The value of the error function $f(\cdot)$ is shown in Fig. 3. It can be seen that the error is exponentially reduced by iteration. Figure 2 shows that the values are well converged to the true values.

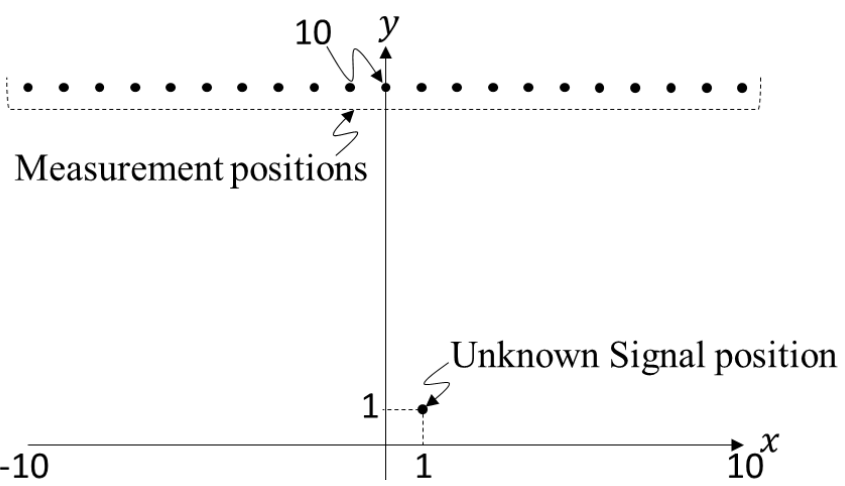

Fig. 1. An unknown signal position and measurement points.

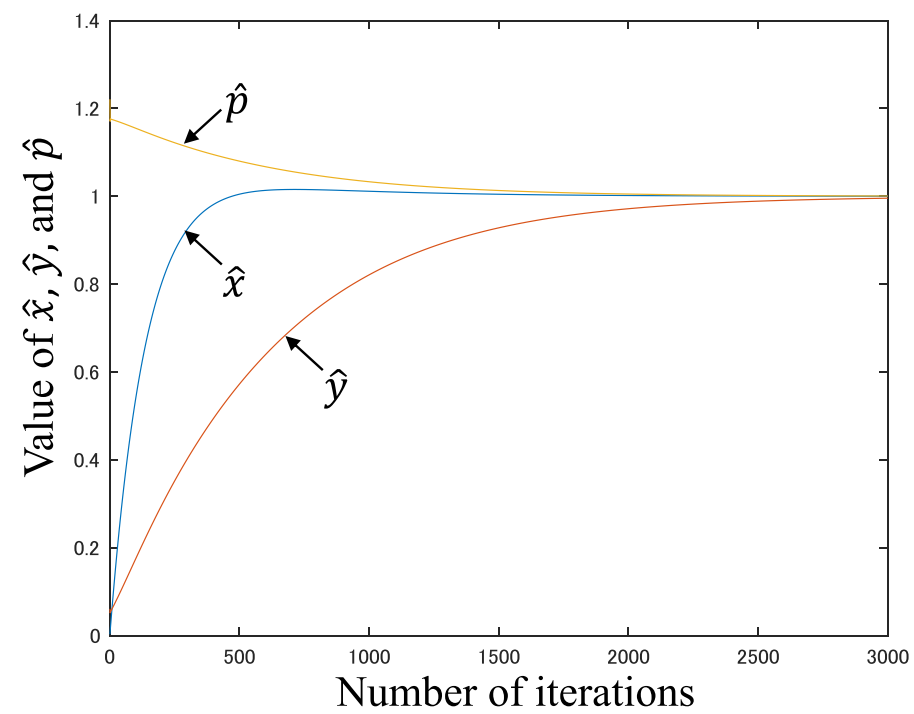

Fig. 2. Simulation results. 


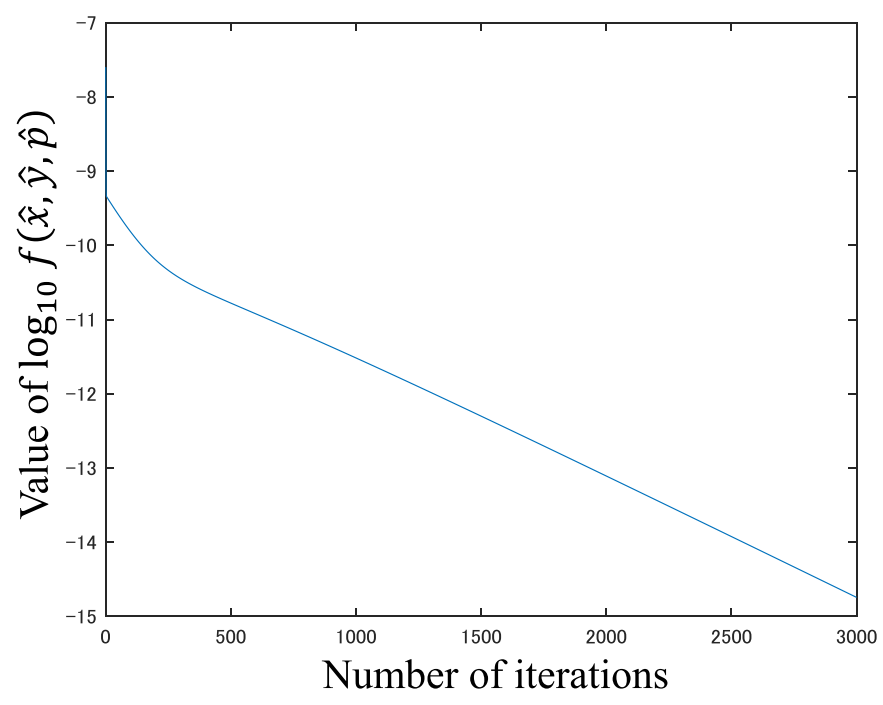

Fig. 3. Value of the error function $f(\cdot)$.

\section{Conclusion}

We have shown the location estimated method of an unknown signal source from the measured RSSI and location information by a nonlinear optimization technique. This is a very practical method because RSSI and location information can be easily obtained with a simple receiver and GPS. We have already developed an extended algorithm when the number of signal sources is unknown. We plan to proceed the verification while conducting actual measurements.

\section{Acknowledgments}

This work is supported by the Ministry of Internal Affairs and Communications under a grant entitled "Research and Development of Optimum Communication Method corresponding to Radio Wave Environment." 